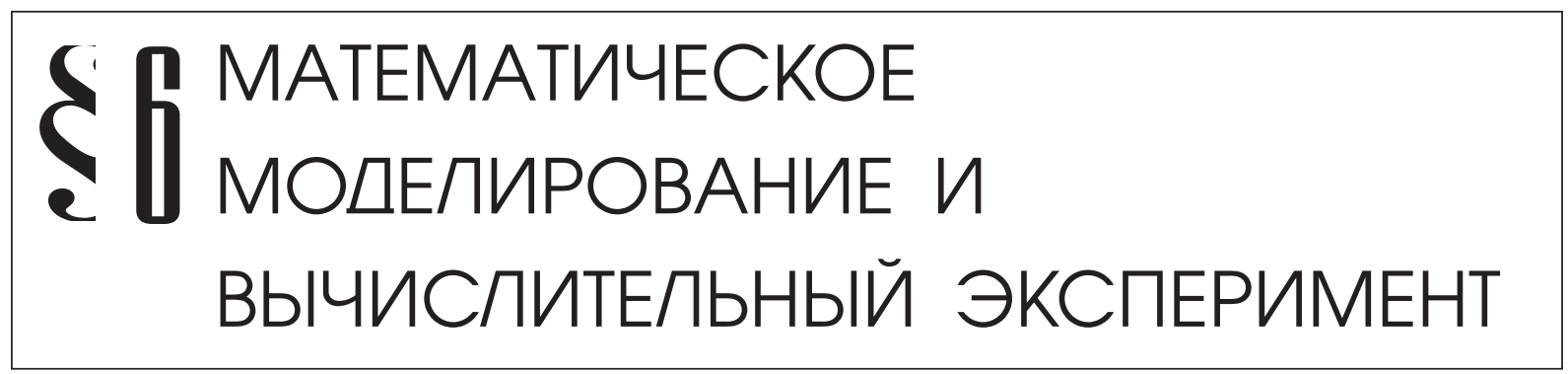

Олейникова С. А.

\title{
РЕКУРСИВНЫЙ ЧИСЛЕННЫЙ МЕТОД ДЛЯ ЭКСПЕРИМЕНТАЛЬНОЙ ОЦЕНКИ ЗАКОНА РАСПРЕДЕЛЕНИЯ ДЛИТЕЛЬНОСТИ ПРОЕКТА В ЗАДАЧАХ СЕТЕВОГО ПЛАНИРОВАНИЯ И УПРАВЛЕНИЯ
}

Аннотация: Рассматривается задача сетевого планирования и управления со случайной длительностью выполнения отдельных операций. Предметом исследования является закон распределения случайной величины, описывающей время выполнения данного проекта. Целью работы является оченка такого закона. Актуальность данной задачи связана с необходимостью повышения точности известных существующих оценок, не принимающих во внимание специфику закона распределения отдельных работ, определяющих проект. Основная сложность практического решения данной задачи заключается в необходимости вычисления кратного определенного интеграла, причем количество отдельных интегралов заранее неизвестно и определяется числом работ, составляющих критический путь данного проекта. В результате предложен численный метод, основанный на рекурсии, который позволяет численно оченить искомый закон распределения. Научная новизна результатов заключается в получении оценок закона распределении длительности проекта, отличающихся повышенной точностью по сравнению с существующими аналогами. Без ограничения общности, разработанный рекурсивный алгоритм может быть использован для широкого класса задач, в которых неизвестно распределение суммы случайных величин при известных распределениях отдельных слагаемых (при предположении о распределении всех значений случайных величин внутри некоторого интервала ограниченной длины).

Ключевые слова: вероятностно-временные характеристики, длительность проекта, закон распределения, бета-распределение, сумма бета-величин, управление проектами, математическая модель рисков, PERT, рекурсия, численный метод 


\section{1. Особенности существующей математической модели рисков, связанных с несвоевременным завершением проекта}

Рассматривается задача составления расписания в случае, если проект задан последовательностью взаимно-зависимых работ со случайной длительностью своего выполнения. В этом случае длительность всего проекта также является случайной величиной. Важной задачей является определение числовых характеристик данной величины, поскольку они являются ключевыми значениями при формировании план-графика. Однако, не менее важными являются задачи оценки разного рода рисков при планировании: вероятность завершения проекта к данному сроку, вероятность того, что проект будет выполнен в заданном временном интервале и т.д. Поскольку невыполнение проекта в срок может привести к различного рода штрафным санкциям или другим нежелательным последствиям, наличие математического аппарата и программного обеспечения, позволяющего с максимально возможной точностью оценить риски при планировании, является весьма актуальной и практически значимой задачей. В частности, для расчета вероятностных характеристик, позволяющих оценить отклонение времени завершения проекта от заданных нормативов, необходимы формулы [1]:

$$
\begin{aligned}
& P(\mathrm{a}<\xi<\mathrm{b})=\int_{a}^{b} f_{\xi}(x) d x=F_{\xi}(b)-F_{\xi}(a) ; \\
& P(\xi<T)=\int_{\min }^{T} f_{\xi}(x) d x=F_{\xi}(T) ; \\
& P(\xi>T)=1-P(\xi<T) .
\end{aligned}
$$

Здесь $F_{\xi}(x)$ - функция распределения случайной величины, описывающей длительность проекта; $f_{\xi}(x)$ - плотность распределения этой величины; $a, b$ и $T$ - заданные пользователем параметры.

Формулы (1), (2) и (3) и определяют математическую модель, которая будет использоваться для оценки рисков [2]. Как видно из этих формул, знание закона распределения является необходимым для оценки рисков, связанных с несвоевременным выполнением проекта.

В настоящее время наиболее известным методом решения данной задачи является метод PERT (Program Evaluation and Review Technique) [3, 4]. Он оценивает исследуемую случайную величину нормальным законом распределения.

Предположение о нормальном распределении длительности проекта базируется на центральной предельной теореме, утверждающей, что сумма бесконечно большого числа одинаково распределенных случайных величин будет распределена нормально. Таким образом, для использования данного закона в виде аппроксимации необходимо наличие двух предпосылок [5,6]:

- число работ, стоящих на критическом пути (т.е. число случайных величин, определяющих длительность проекта) должно быть очень велико; 
- все случайные величины, определяющие отдельные работы, должны быть распределены нормально.

Очевидно, что в реальных условиях ни одно из этих утверждений выполняться не будет. В связи с этим, можно предположить, что существует другой закон, позволяющий оценить длительность проекта с меньшей погрешностью.

Для обоснования необходимости других подходов к оценке длительности проекта был проведен следующий вычислительный эксперимент [7]. На вход подавалось количество случайных величин, которые будут суммироваться, а также параметры каждой из этих величин. На выходе была получена выборка, каждый элемент которой представлял собой сумму заданного числа бета-величин с заданными параметрами распределения. Результаты, представленные в виде гистограмм, свидетельствовали о необходимости подбора более точной аппроксимации, чем нормальный закон распределения. В частности, была выдвинута гипотеза о возможной аппроксимации суммы бета-величин бета-распределением. Для экспериментального подтверждения такой гипотезы необходим численный метод, который с заданной точностью может аппроксимировать сумму бета-величин.

\section{2. Общий подход к реализации численного метода решения}

Исходя из поставленной задачи, разработаем общий подход к ее решению. Специфика формулы бета-распределения обуславливает необходимость поиска численного подхода к определению плотности итоговой случайной величины.

Формула плотности распределения суммы двух случайных величин имеет следующий вид [8]:

$$
f_{\eta}(z)=\int_{-\infty}^{\infty} f_{\xi_{1}}(x) \cdot f_{\xi_{2}}(z-x) d x .
$$

Выведем рекурсивную формулу, позволяющую найти плотность от суммы n случайных величин [9].

Пусть

$$
\eta_{n}=\xi_{1}+\ldots+\xi_{n}=\eta_{n-1}+\xi_{n},
$$

Где

$$
\eta_{n-1}=\xi_{1}+\ldots+\xi_{n-1} .
$$

Тогда

$$
f_{\eta_{n}}(x)=\int_{-\infty}^{\infty} f_{\eta_{n-1}}\left(x_{n-1}\right) \cdot f_{\xi_{n}}\left(x-x_{n-1}\right) d x_{n-1} .
$$

Аналогичным образом можно описать плотность распределения случайной величины $\eta_{\mathrm{n}-1}$ :

$$
\eta_{n-1}=\xi_{1}+\ldots+\xi_{n-2}+\xi_{n-1}=\eta_{n-2}+\xi_{n-1}
$$


Тогда

$$
f_{\eta_{n-1}}(x)=\int_{-\infty}^{\infty} f_{\eta_{n-2}}\left(x_{n-2}\right) \cdot f_{\xi_{n-1}}\left(x-x_{n-2}\right) d x_{n-2} .
$$

Продолжая рассуждения аналогичным образом, на последнем шаге получим следующую случайную величину:

$$
\eta_{2}=\eta_{1}+\xi_{2}
$$

Где

$$
\eta_{1}=\xi_{1}
$$

Таким образом, получили конечную точку разложения:

$$
\eta_{2}=\xi_{1}+\xi_{2},
$$

для которой можно в явном виде определить плотность:

$$
f_{\eta_{2}}(x)=\int_{-\infty}^{\infty} f_{\xi_{1}}\left(x_{1}\right) \cdot f_{\xi_{2}}\left(x-x_{1}\right) d x_{1} .
$$

Таким образом, можно разработать рекурсивный алгоритм, который n-кратный интеграл, описанный с помощью формулы (7) сведет к $n$-кратному вызову подпрограммы, вычисляющей на каждом шаге интеграл от суммы меньшего числа слагаемых [10]. Для выхода из рекурсии будет использоваться формула (10), которая позволяет рассчитать интеграл уже в явном виде, поскольку предполагается, что плотности распределения отдельных слагаемых известны. Для вычисления такого интеграла можно воспользоваться любым численным методом интегрирования [11,12].

Исходя из описанных рассуждений, выведем общий вид рекурсивного алгоритма, позволяющего с заданной точностью определить закон распределения случайной величины, описывающей длительность проекта:

Исходные данные: множество работ $W$, стоящих на критическом пути.

Шаг 1. Если количество работ $n=2$, то определить интеграл по формуле (10)

Иначе перейти к шагу 2.

Шаг 2. Воспользоваться для вычисления интеграла формулой (4).

\section{3. Детализация алгоритма с учетом бета-распределения длительностей отдельных работ}

Рассмотрим более подробно реализацию предложенного выше алгоритма в предположении о бета-распределении отдельных работ, входящих в проект. Общий вид плотности бета-распределения имеет вид [13]:

$$
f_{\xi}(t)=\left\{\begin{array}{l}
\frac{(t-a)^{p-1}(b-t)^{q-1}}{(b-a)^{p+q-1} B(p, q)}, a \leq t \leq b \\
0, t<a, t>b .
\end{array}\right.
$$


Здесь $[a, b]$ - интервал распределения; $p$ и $q$ - параметры распределения. В этом случае несложно показать, что случайная величина $\eta_{n}$, определенная формулой (5), будет распределена в интервале $\left[a_{1}+\ldots+a_{n}, b_{1}+\ldots+b_{n}\right]$; случайная величина $\eta_{n-1}$ - в интервале $\left[a_{1}+\ldots+a_{n-1}, b_{1}+\ldots+b_{n-1}\right]$ и т.д. С учетом этих интервальных ограничений плотности распределения, определим пределы интегрирования на каждом шаге.

Введем следующие обозначения. Пусть переменная $l$ представляет собой левую границу области определения текущей случайной величины, а $r$ - правую границу. Изначально

$$
\begin{aligned}
& l=a_{1}+\ldots+a_{n} ; \\
& r=b_{1}+\ldots+b_{n} .
\end{aligned}
$$

Очевидно, что интеграл (7) имеет смысл вычислять лишь при ненулевых значениях плотности. Следовательно, обе плотности должны быть ненулевыми. Первый множитель представляет собой плотность распределения случайной величины $\eta_{n-1}$. Левая граница данной плотности будет определена путем вычета из левой границы предыдущей случайной величины ее нижнего значения:

$$
l_{\text {нов }}=l_{\text {стар }}-a_{n} ;
$$

а правая - путем вычета верхнего значения:

$$
r_{\text {нов }}=r_{\text {стар }}-b_{n} \text {. }
$$

Второй множитель - это плотность распределения случайной величины $\xi_{n}$. Согласно формуле (8), она распределена в интервале $\left[a_{n}, b_{n}\right]$. Пусть $x$ - это аргумент искомой плотности, $t$ - это текущая переменная интегрирования. Тогда второй множитель будет неотрицателен при выполнении следующих неравенств:

$$
\left\{\begin{array}{l}
x-t \geq a \\
x-t \leq b
\end{array}\right. \text {. }
$$

С учетом ограничений (14)-(16), можно предложить следующий алгоритм рекурсивного численного метода определения плотности суммы $\mathrm{n}$ бета-величин, представленный на рисунке 1.

Рассмотрим теперь нерекурсивную ветвь алгоритма, связанную с вычислением интеграла по формуле (10). В данном случае подынтегральное выражение представляет собой произведение двух плотностей: для первого и второго слагаемых. Как видно из формулы (10), аргумент первой функции - это переменная интегрирования. Следовательно, он должен изменяться в следующих пределах:

$$
a_{1} \leq t \leq b_{1} \text {. }
$$

Здесь $t$ - переменная интегрирования.

Второй множитель - это плотность второго слагаемого с аргументом $x$ - $t$, где $x$ - аргумент функции, а $t$ - переменная интегрирования. Для нее необходимо выполнение 
следующих неравенств:

$$
\left\{\begin{array}{l}
x-t \geq a_{2} \\
x-t \leq b_{2}
\end{array} .\right.
$$

Таким образом, рекурсивный алгоритм оценки закона распределения будет иметь вид, представленный на рисунке 1.

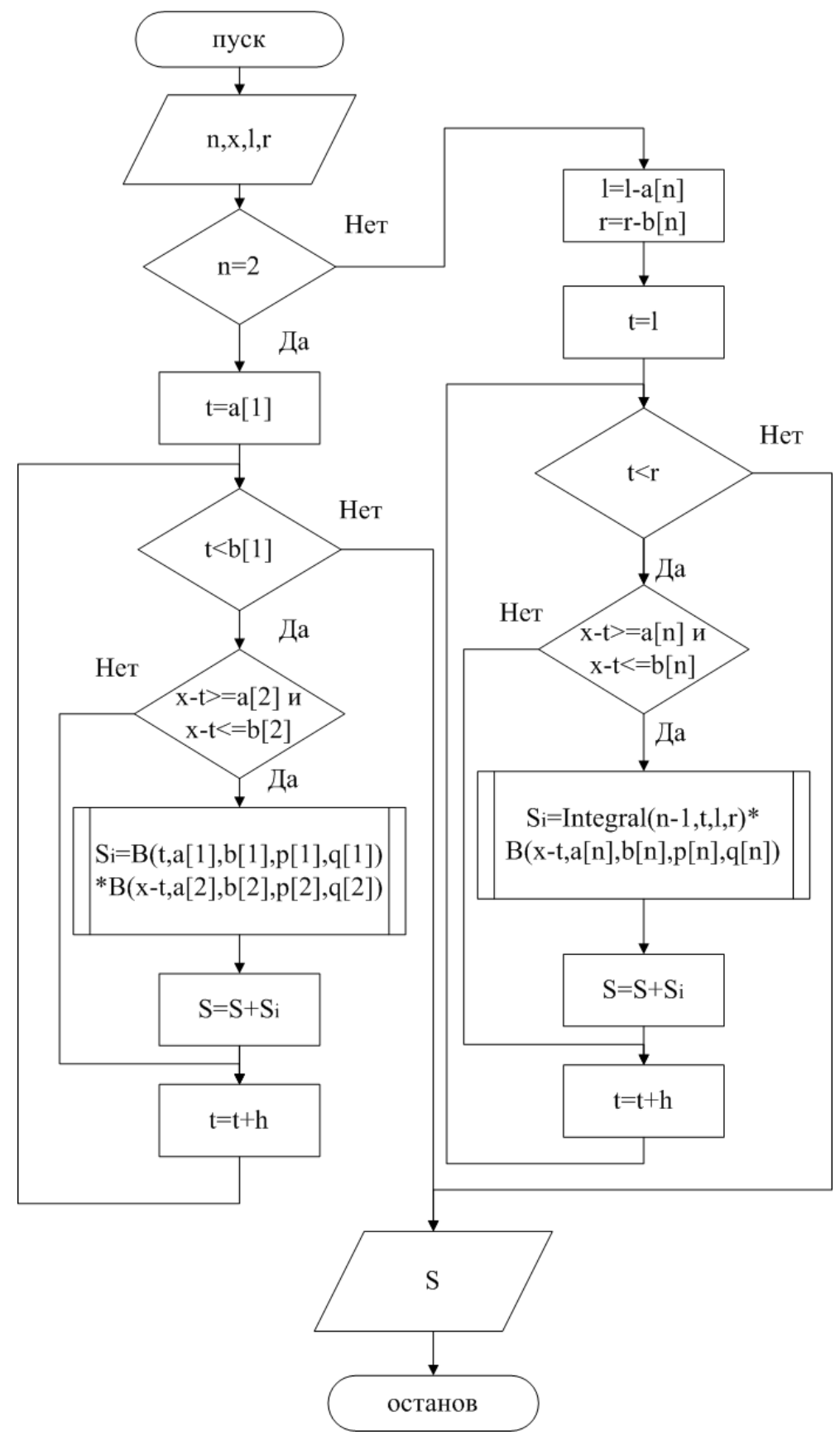

Рисунок 1 - Алгоритм рекурсивного численного метода 
Приведем пояснения к данному алгоритму. Здесь $n$ - количество бета-величин (слагаемых); $x$ - аргумент, для которого необходимо найти закон распределения; $l$ и $r$ - левая и правая границы области определения закона; $a[i], b[i], p[i], q[i]$ - массивы размера $n$, которые хранят параметры бета-величин; $B(\arg , a, b, p, q)$ - плотность бета-распределения для случайной величины с параметрами $a, b, p$ и $q$, рассчитанная для аргумента arg (формула (11)); Integral - рекурсивный вызов данной функции; $h$ - шаг интегрирования; $S$ - переменная - площадь фигуры (геометрический смысл интеграла).

\section{4. Выводы}

Проанализируем практическую значимость полученных результатов. Без ограничения общности, разработанный рекурсивный алгоритм может быть использован для широкого класса задач, в которых неизвестно распределение суммы случайных величин при известных распределениях отдельных слагаемых (при предположении о распределении всех значений случайных величин внутри некоторого интервала ограниченной длины) [14-19]. В данном случае алгоритм разрабатывался из-за необходимости наличия закона распределения случайной величины, описывающей длительность проекта, для решения задач сетевого планирования и управления. Знание данного закона позволит оценить вероятностно-временные характеристики случайной величины, описывающей длительность проекта, с помощью математической модели (1)-(3), и, следовательно, оценить риски, связанные с возможной задержкой его выполнения.

Таким образом, можно сделать следующие выводы.

1. Обоснована целесообразность решения данной задачи, исходя из ее детального анализа, а также базируясь на экспериментальных данных, подтверждающих необходимость улучшения существующих оценок.

2. Описан общий подход для численной оценки плотности распределения суммы случайных величин. Он представляет собой рекурсивную схему, на каждом шаге которой есть возможность перехода к сумме меньшего числа слагаемых.

3. Детализирован алгоритм оценки закона распределения для суммы случайных величин при условии, что они распределены по закону бета.

Данный алгоритм может применяться для широкого класса задач, в которых необходимо исследование законов распределения суммы некоторых случайных величин. В данной работе показано применение результатов для класса задач сетевого планирования и управления, где наличие такого закона необходимо для оценки рисков, связанных с несвоевременным завершением проектов.

\section{Библиография :}

1. Вентцель Е.С. Теория вероятностей. - М. Физматлит, 1962. - $564 c$. 
2. Олейникова С.А. Математическая модель и оптимизационная задача составления расписания для мультипроектной системы с временными и ресурсными ограничениями и критерием равномерной загрузки// Вестник Воронежского государственного технического университета. 2013. Т. 9. № 6-3. С. 58-61.

3. Олейникова С.А. Критический анализ метода PERT решения задачи управления проектами со случайной длительностью выполнения работ// Системы управления и информационные технологии. № 1(51), 2013. - с. 20-24. Кобзарь А.И. Прикладная математическая статистика. Для инженеров и научных работников. М.: Физматлит, 2006. -816 с.

4. WILLIAMS, T. M. (1995) What are PERT estimates? J. Oper. Res. Soc., 46, 1498-1504.

5. Олейникова С.А. Оценка критического времени в задачах управления проектами// Вестник Воронежского государственного технического университета. 2011. Т. 7. № 2. С. 106-109.

6. Олейникова С.А. Численная оценка времени обслуживанияв задачахсетевого планирования и управления// Вестник Воронежского государственного технического университета. 2009. Т. 5. № 3. С. 111-114.

7. Олейникова С.А. Вычислительный эксперимент для анализа закона распределения случайной величины, описывающей длительность проекта в задачах сетевого планирования и управления// Экономика и менеджмент систем управления, 2013. Т.9. № 3. с. 90-96.

8. Кобзарь А.И. Прикладная математическая статистика. Для инженеров и научных работников. - М.: Физматлит, 2006. $-816 \mathrm{c}$.

9. Пирогов А.М., Олейникова С.А. $0 б$ одном подходе к оценке длительности проекта в задачах сетевого планирования и управления// Информационные технологии в вычислительной техникеи связи: Материалы II Межд. конф. Выпуск ІІ.-Воронеж, Международный институт компьютерных технологий, 2013. - с. 57-69.

10. Мальцев А.И. Алгоритмы и рекурсивные функции. - М., Наука, Гл. ред. физ.-мат. лит. 1986. - 368 с.

11. Крылов В.И., Бобков В.В., Монастырский П.И. Начала теории вычислительных методов. Интерполирование и интегрирование. Минск, Наука и техника, 1983. - 287с.

12. Вержбицкий В.М. Основы численных методов. М.: Высш. шк., 2005. - 840с.

13. Wise M.E. The incomplete beta-function as a contour integral and a quickly conversing series for its inverse // Biometrika. 1950. V. 37. P. 208-218.

14. Лабковская Р.Я., Козлов А.С., Пирожникова О.И., Коробейников А.Г. Моделирование динамики чувствительных элементов герконов систем управления // Кибернетика и программирование.-2014.-5.-С. 70-77. D0I: 10.7256/2306-4196.2014.5.13309. URL: http://www.e-notabene.ru/kp/article_13309.html

15. Л. С. Кирина Этапы управления налоговыми рисками в налоговом консультировании // Налоги и налогообложение.-2012.-5.-С. 46-54.

16. Гракова Н.В. Построение семантической модели управления проектами // Кибернетика и программирование.-2012.-1.-C. 7-15. URL: http://www.e-notabene.ru/kp/article_13857.html

17. Клименко А.Б., Троценко Р.В. Решение задачи оптимизации ресурсов и планирования вычислений с использованием параллельной имитации отжига // Программные системы и вычислительные методы.2014.-3.-C. 282-290. D0I: 10.7256/2305-6061.2014.3.13419.

18. Новакова Н.Е., Горячев А.В., Горячев А.А., Васильев А.А., Монахов А.В. Система управления проектами В автоматизированном проектировании // Кибернетика и программирование.-2013.-4.-С. 1-13. DOI: 10.7256/2306-4196.2013.4.8301.URL: http://www.e-notabene.ru/kp/article_8301.html 
19. Комарцова Л.Г., Лавренков Ю.Н., Антипова О.В. Комплексный подход к исследованию сложных систем // Программные системы и вычислительные методы.-2013.-4.-C. 330-334. DOI: 10.7256/23056061.2013.4.10551.

\section{References:}

1. Venttsel'E.S. Teoriya veroyatnostei. - M. Fizmatlit, 1962. -564 s.

2. Oleinikova S.A. Matematicheskaya model'i optimizatsionnaya zadacha sostavleniya raspisaniya dlya mul'tiproektnoi sistemy s vremennymi i resursnymi ogranicheniyami i kriteriem ravnomernoi zagruzki// Vestnik Voronezhskogo gosudarstvennogo tekhnicheskogo universiteta. 2013. T. 9. № 6-3. S. 58-61.

3. Oleinikova S.A. Kriticheskii analiz metoda PERT resheniya zadachi upravleniya proektami so sluchainoi dlitel'nost'yu vypolneniya rabot// Sistemy upravleniya i informatsionnye tekhnologii. № 1(51), 2013. - s. 20-24. Kobzar' A.I. Prikladnaya matematicheskaya statistika. Dlya inzhenerov i nauchnykh rabotnikov. - M.: Fizmatlit, 2006. - $816 \mathrm{~s}$.

4. WILLIAMS, T. M. (1995) What are PERT estimates? J. Oper. Res. Soc., 46, 1498-1504.

5. Oleinikova S.A. Otsenka kriticheskogo vremeni v zadachakh upravleniya proektami// Vestnik Voronezhskogo gosudarstvennogo tekhnicheskogo universiteta. 2011. T. 7. № 2. S. 106-109.

6. Oleinikova S.A. Chislennaya otsenka vremeni obsluzhivaniya v zadachakh setevogo planirovaniya i upravleniya// Vestnik Voronezhskogo gosudarstvennogo tekhnicheskogo universiteta. 2009. T. 5. № 3. S. 111-114.

7. Oleinikova S.A. Vychislitel'nyi eksperiment dlya analiza zakona raspredeleniya sluchainoi velichiny, opisyvayushchei dlitel'nost' proekta v zadachakh setevogo planirovaniya i upravleniya// Ekonomika i menedzhment sistem upravleniya, 2013. T.9. № 3. s. 90-96.

8. Kobzar' A.I. Prikladnaya matematicheskaya statistika. Dlya inzhenerov i nauchnykh rabotnikov. - M.: Fizmatlit, 2006. $-816 \mathrm{~s}$.

9. Pirogov A.M., Oleinikova S.A. Ob odnom podkhode k otsenke dlitel'nosti proekta v zadachakh setevogo planirovaniya i upravleniya// Informatsionnye tekhnologii v vychislitel'noi tekhnike i svyazi: Materialy II Mezhd. konf. Vypusk II.-Voronezh, Mezhdunarodnyi institut komp'yuternykh tekhnologii, 2013. - s. 57-69.

10. Mal'tsev A.I. Algoritmy i rekursivnye funktsii. - M., Nauka, Gl. red. fiz.-mat. lit. 1986. - 368 s.

11. Krylov V.I., Bobkov V.V., Monastyrskii P.I. Nachala teorii vychislitel'nykh metodov. Interpolirovanie i integrirovanie. Minsk, Nauka i tekhnika, 1983. - 287s.

12. Verzhbitskii V.M. Osnovy chislennykh metodov. M.: Vyssh. shk., 2005. - 840s.

13. Wise M.E. The incomplete beta-function as a contour integral and a quickly conversing series for its inverse // Biometrika. 1950. V. 37. P. 208-218.

14. Labkovskaya R.Ya., Kozlov A.S., Pirozhnikova 0.I., Korobeinikov A.G. Modelirovanie dinamiki chuvstvitel'nykh elementov gerkonov sistem upravleniya // Kibernetika i programmirovanie.-2014.-5.-C. 70-77. D0I: 10.7256/23064196.2014.5.13309. URL: http://www.e-notabene.ru/kp/article_13309.html

15. L. S. Kirina Etapy upravleniya nalogovymi riskami v nalogovom konsul'tirovanii // Nalogi i nalogooblozhenie.2012.-5.-C. 46-54.

16. Grakova N.V. Postroenie semanticheskoi modeli upravleniya proektami // Kibernetika i programmirovanie.-2012.1.-C. 7-15. URL: http://www.e-notabene.ru/kp/article_13857.html 
17. Klimenko A.B., Trotsenko R.V. Reshenie zadachi optimizatsii resursov i planirovaniya vychislenii s ispol'zovaniem parallel'noi imitatsii otzhiga // Programmnye sistemy i vychislitel'nye metody.-2014.-3.-C. 282-290. D0I: 10.7256/2305-6061.2014.3.13419.

18. Novakova N.E., Goryachev A.V., Goryachev A.A., Vasil'ev A.A., Monakhov A.V. Sistema upravleniya proektami v avtomatizirovannom proektirovanii // Kibernetika i programmirovanie.-2013.-4.-C. 1-13. D0I: 10.7256/23064196.2013.4.8301.URL: http://www.e-notabene.ru/kp/article_8301.html

19. Komartsova L.G., Lavrenkov Yu.N., Antipova 0.V. Kompleksnyi podkhod k issledovaniyu slozhnykh sistem // Programmnye sistemy i vychislitel'nye metody.-2013.-4.-C. 330-334. D0I: 10.7256/2305-6061.2013.4.10551. 